\title{
Arrayed wide-field astronomical camera system for spectroscopic surveys on Extremely Large Telescopes: system architecture, proof-of-concept, and enabling technologies
}

\author{
Hanshin Lee $\odot^{\mathrm{a}, *}$ and Menelaos K. Poutous ${ }^{\mathrm{b}}$ \\ ${ }^{a}$ University of Texas at Austin, McDonald Observatory, Austin, Texas, United States \\ ${ }^{b}$ University of North Carolina, Department of Physics and Optical Sciences, Charlotte, \\ North Carolina, United States
}

\begin{abstract}
The next generation Extremely Large Telescopes (ELTs) are promising a profound transformation of humanity's understanding of the universe by opening our eyes to a myriad of previously unseen astronomical objects across cosmic space time. Some of the key observational contributions to this transformation will, once again, be made through large-scale ultradeep spectroscopic surveys. Such surveys will call for a wide-field system to expand field of view and correct atmospheric dispersion beyond what an uncorrected ELT is canonically capable of. Although the traditional monolithic form of field correctors served our needs very well on 2- to 8-m class telescopes, we recognize challenges around scaling this traditional design to the ELT level, and hence, as detailed here, present a fundamentally different architecture, called the arrayed wide-field astronomical corrector system or AWACS, for the ELTs of two-mirror construction. The AWACS accomplishes field expansion via an array of small units populated over a telescope's focal surface, compensating for field aberrations and atmospheric dispersion locally but simultaneously. The AWACS units share one common electro-opto-mechanical design, permitting cost-effective high-volume part manufacturing. We detail the architectural features and proof-of-concept on-sky demonstration of the AWACS. In addition, we highlight our recent development results of randomly nano-textured antireflective (AR) surface structures in terms of an immediately viable, super-broadband, high-performance AR solution for not only the AWACS optics, but also broader ranges of electro-optical devices, particularly those subjected to harsh environments such as high-power laser, cryogenic, and space systems. With continuous advances in other relevant fields, the AWACS is uniquely positioned to enable, either by itself or by complementing traditional correctors, wide-field multi-object spectroscopic surveys in the ELT era and beyond. () The Authors. Published by SPIE under a Creative Commons Attribution 4.0 Unported License. Distribution or reproduction of this work in whole or in part requires full attribution of the original publication, including its DOI. [DOI: 10.1117/1.JATIS.7.3.035007]
\end{abstract}

Keywords: arrayed wide-field astronomical corrector system; wide-field corrector; extremely large telescopes; spectroscopy; survey.

Paper 21038 received Mar. 30, 2021; accepted for publication Jul. 27, 2021; published online Aug. 19, 2021.

\section{Introduction}

The next decade will see the arrival of three 30-m class Extremely Large Telescopes (ELTs). ${ }^{1-3}$ These giants are promising a profound transformation in humanity's understanding of the universe by opening our eyes to the unprecedented details of a myriad of astronomical objects. As we consider some of the pivotal roles played by astronomical surveys in the past, ${ }^{4-6}$ it seems reasonable to expect that such a new transformation will, once again, be driven by much more detailed large-scale spectroscopic surveys of extremely faint astronomical objects at different physical scales across cosmic space time. Some of the obvious beneficiaries from such surveys include galactic archaeology (i.e., chemical tagging) on Milky Way stars down to the faintest limits that the ELTs can offer, ${ }^{1-3,7,8}$ accurate mapping of low-mass/density structure formation

*Address all correspondence to Hanshin Lee, lee@astro.as.utexas.edu 
via ubiquitous yet extremely faint dwarf galaxies in the local universe, ${ }^{1-3}$ and improving constraints of the time evolution and spatial inhomogeneity of reionization of the universe via surveying Lyman-alpha galaxies at high redshifts. ${ }^{9-11}$ But many will surely find ingenious ways to utilize such survey instruments and data for originally unintended yet intriguing scientific endeavors fueled by new theories and observations as we frequently witnessed in the past.

In an ideal world, one would like to get the most out of these ELT-class surveys per any given night and insist on running them at the highest possible efficiency. One would also expect such surveys to produce scientific data products in unprecedented sensitivity across a broad wavelength range. Potentially, this would call for, on a single ELT, wide-field access by multiple back-end bench-mounted spectrographs, all operating simultaneously and possibly in different spectral regions, resolutions, and on different targets. Such a "parallel" observation as previously described ${ }^{12}$ is in full operation [e.g., at the Hobby-Eberly telescope (HET)] and, at minimum, its application to the ELTs will call for a wide field of view (FOV) and atmospheric dispersion correction (ADC) beyond what these uncorrected telescopes are canonically capable of.

Looking back, we recognize that astronomical correctors traditionally consist of 3 to 8 large monolithic meniscus lenses ${ }^{13,14}$ plus a set of ADC prisms (except the cases of the HET ${ }^{15}$ and the South African Large Telescope ${ }^{16}$ where field correctors are comprised of mirrors), all following completely one-off construction process. Although this approach did work, scaling it to larger and larger aperture telescopes inevitably resulted in field correctors with greater volume, complexity, and cost. It should come as no surprise that these factors resulted in the immensity and complexity of monolithic correctors on existing 8- to 10-m telescopes, while calling for optical elements and thin-film coatings often considered beyond the state-of-the art with astronomical price tags. ${ }^{14}$ This may be a harbinger of challenges in scaling such a traditional field corrector to the ELT level that we must pay attention to ahead of time.

Having said that we emphasize that our focus here is not so much about yet-to-be-substantiated pessimism toward the ELT-class field correctors in some traditional form, but rather about more fundamental questions such as, "Does the ELT wide-field system have to be in a traditional monolithic form?"; "What alternative field corrector architecture is plausible?"; "Do we have necessary technologies to turn this new architecture into a reality?" In our effort to address these questions, we explored an imaging architecture that is fundamentally orthogonal to the traditional design, called the Arrayed Wide-field Astronomical Corrector System or AWACS, mainly for the ELTs of two-mirror construction, such as the Giant Magellan telescope (GMT) ${ }^{1}$ and the Thirty Meter telescope. ${ }^{2}$

Not surprisingly, we were not the first to try to answer these questions. Burge and Angel ${ }^{17}$ considered a two-mirror off-axis relay concept that locally corrects mainly spherical aberration at the image plane of a two-mirror telescope with a 30-m spherical primary. Each relay unit had a limited (a few arc-second wide) field, mainly aiming for feeding a fiber or small integral-field unit (IFU) but demonstrated what could be accomplished with an "array" of such relay units. In the same spirit, Ragazzoni, ${ }^{18}$ a year later, reported a similar concept for an ELT, called the smart fast camera, that is based on a refractive relay with an internal aberration corrector for feeding fibers or mini IFUs. Both solutions share the fundamental arrayed architecture, but Ragazzoni's solution more closely mirrors some of the design details of the AWACS, which is independently developed in the presented study.

In essence, the AWACS accomplishes desired field correction via an array of small costeffective electro-opto-mechanical (EOM) relay units populated over a telescope's focal surface. These units compensate for the telescope field aberrations and atmospheric dispersion locally and simultaneously. In some ways, the AWACS is similar to the segmented mirror telescopes with one major difference, that is the AWACS utilizes focal plane segmentation as the overarching scaling mechanism. Also the AWACS heavily relies on commonality of design features across units in order to maximize its scalability. These are discussed in the context of addressing the first two questions above (Section 2). As part of this study, we constructed a dual-unit proofof-concept (POC) AWACS out of commercial optics and off-the-shelf cage mechanics plus 80/20 aluminum extrusions. The POC AWACS has been tested on the 2.7-m Harlan J. Smith telescope (HJST) at the McDonald Observatory during the nights between August 20 
and 26 in 2018. Since the POC units were limited in many aspects, the main test focus was to validate the local field aberration correction using a pair of cylindrical lenses. This on-sky test result is discussed in Sec. 3. In Sec. 4, we focus on the topic of antireflective (AR) treatment of air-glass interfaces. Specifically, we highlight the tendency of the AWACS to require a large number of such interfaces and the paramount importance of high-performance AR treatment of these interfaces. We compare different AR treatment options and pay a special attention to nano-structured optical surfaces as the viable pathway to a super broadband AR solution for the AWACS, thereby addressing the last question above.

\section{AWACS Design Features}

The AWACS is one specific type of arrayed imaging architectures that include segmented mirror telescopes (e.g., the $\mathrm{HET}^{19}$ and the Keck ${ }^{20}$ ), radio telescope arrays (e.g., the very large array ${ }^{21}$ ), and ground surveillance systems such as the DARPA's AWARE system. ${ }^{22}$ Unlike segmented mirror telescopes, the AWACS relies on the focal plane segmentation as the main scaling mechanism. In that sense, it is similar to the AWARE system. Although segmented mirror telescopes aim for smallest intersegment gaps to achieve minimal light loss and point-spread function (PSF) diffraction structures, the focal plane segmentation can likely tolerate larger gaps between adjacent AWACS units because of generally sparse and/or clumpy spatial distributions of astronomical objects. The main priority is the "access to wide-field," which could be enabled, without $100 \%$ instantaneous fill factor, using only a limited number of AWACS units positioned at strategic fields. These units could be later reconfigured over a plug plate with a predetermined grid of discrete ports or on continuous three-dimensional (3D) trajectories in either an open- or closedloop fashion via, for example, pick-and-place robotic arms.

Figure 1 illustrates the main features of the AWACS. Figure 1(a) shows an example monolithic corrector without ADC. Figure 1(b) shows the literally segmented version of (a), which may not be ideal for a field corrector. Figure 1(c) shows the AWACS and Figure 1(d) highlights one possible AWACS configuration with wide-field access to multiple instruments. These may include fiber IFU and/or multi-slit spectrographs, or imaging sensors at different parts of the native and Nasmyth foci. All of these could operate simultaneously or sequentially over different

(a)

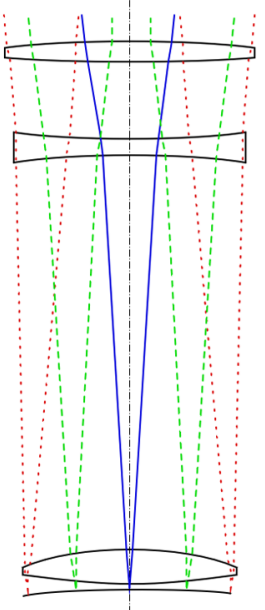

(b)

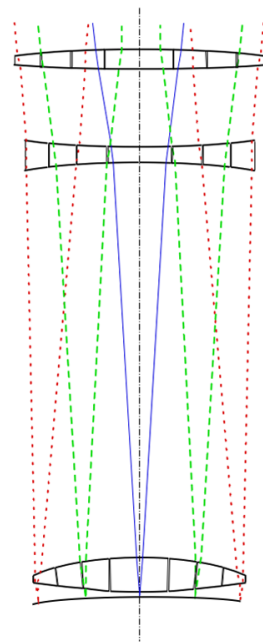

(c)

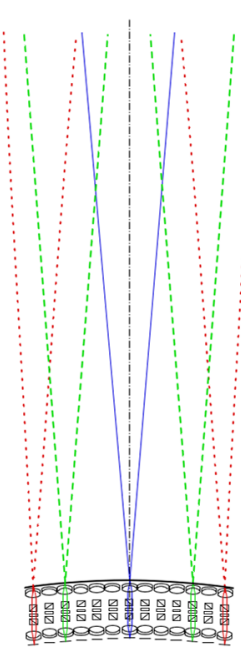

(d)

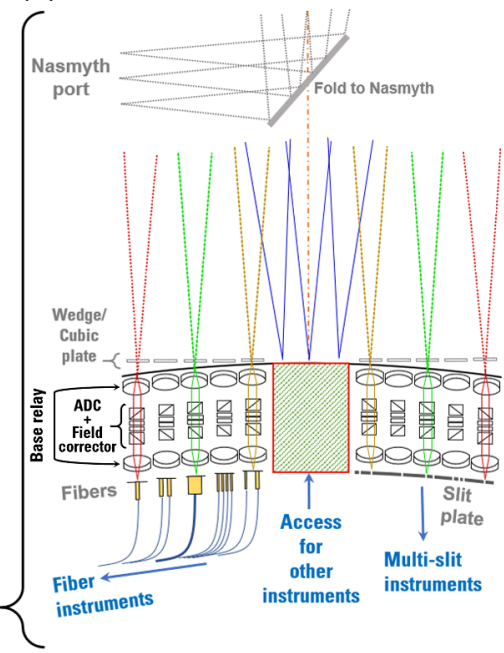

Fig. 1 An illustration of three canonical field corrector architectures. Note that light propagates from top (i.e., telescope) to bottom. (a) Monolithic version without ADC prisms; (b) literally segmented version of (a); (c) relay-based arrayed architecture with ADC prisms and aberration compensator in the central air space of each relay; and (d) close-up of the cross-section view of (c) where a single-wide-field platform either sequentially or simultaneously hosts multiple heterogeneous instruments at different instrument ports (i.e., native annular field, native central field, or Nasmyth central field via a fold mirror). 
parts of the FOV. Note that the degree of needed field correction diminishes near the field center. This portion of the focal surface could be left open for access by instruments to which the impact of uncorrected atmospheric dispersion or field aberration is either deemed tolerable or can be calibrated. Figure 1(c) highlights that the AWACS can be confined to a compact volume behind the native telescope focal surface. Structurally, this could give an opportunity to completely decouple it from the telescope with a clean insertion/retraction path to/from the telescope focus.

\subsection{Canonical Design Features}

For further discussion of the AWACS design features, we focus on Figs. 1(c) and 1(d), which illustrate heavy reliance on design commonality for the AWACS scalability. The goal is that all units are the exact replicas of one single EOM unit design that contains the following canonical design features.

\subsubsection{Base relay and $A D C$ prisms}

First, the unit design features a set of imaging lenses forming the underlying relay. Primarily, it transfers a patch of the native telescope focus to the instrument input with minimal additional optical aberrations. The relay magnification can be application-specific. These imaging lenses can be made identical across all units at different fields, allowing the replication of one common optomechanical packaging. This makes the base relay largely agnostic about the telescope's aberration characteristics, giving the opportunity to be adaptable to different telescopes as long as the relay focal ratio and FOV are suitable on those telescopes.

The second feature, which is located in the internal collimated air space, is a pair of identical prism doublets. This pair performs the function of ADC with the well-known working mechanism as follows. Each doublet is a Risley prism made of two prism singlets bonded to each other, one made from a high-dispersion glass material and the other from a low-dispersion material. ${ }^{23}$ The counter-rotation angle $(\omega)$ between the dispersion axes of these prism doublets determines the corrective power (e.g., full compensation at $\omega=0$ deg and no compensation at $\omega=180 \mathrm{deg}$ or vice versa). $\omega$ can be tuned to compensate for the atmospheric dispersion at a specific zenith angle $(Z)$.

One detail to keep in mind is that, due to the Lagrange-Helmholtz invariant (also known as conservation of étendue or $A \Omega$ product), the differential refraction angle on sky is scaled at the ADC prism by the ratio between the telescope focal length $f_{\text {tel }}$ and that of the AWACS base relay $f_{\text {rel }}$. For example, with the same $f_{\text {rel }}$, the ADC prisms need higher angular dispersion on a larger telescope (i.e., longer $f_{\text {tel }}$ ) for the same amount of atmospheric dispersion that is otherwise correctable using less dispersive prisms on a smaller telescope (i.e., shorter $f_{\text {tel }}$ ). This requires either steeper prism angles and/or larger dispersion index difference between prism glass materials.

Figure 2 shows the variation of the differential refraction across five zernith angle offsets around $Z=60 \mathrm{deg}$ based on the standard model. ${ }^{24}$ Each differential refraction angle curve is given relative to that of wavelength $5000 \AA$. The variation between them shows practically constant variation of the dispersion across the FOV expected for the ELTs in their wide-field mode. Of course, it is possible to knock out even the field dependent residual dispersion by locally tuning $\omega$ for different AWACS units. However, the model mismatch from the actual atmospheric dispersion at a given site is likely more dominant source of error. Hence, for all practical purposes, the prisms can be electro-opto-mechanically uniform, allowing one ADC assembly packaging, electronics, and control solution be replicated.

\subsubsection{Cylinder lens pair}

In addition to the ADC prisms, the collimated air space contains another feature, a pair of cylinder lenses. This pair forms the field aberration corrector, more specifically for field astigmatism. This choice is simply due to the field astigmatism being the dominant field aberration of modern two-mirror aplanatic telescopes. ${ }^{23}$ The pair consists of one positive and one negative cylinders and their sequence in the direction of optical path can be arbitrary.

J. Astron. Telesc. Instrum. Syst. $\quad$ 035007-4 Jul-Sep 2021 • Vol. 7(3) 


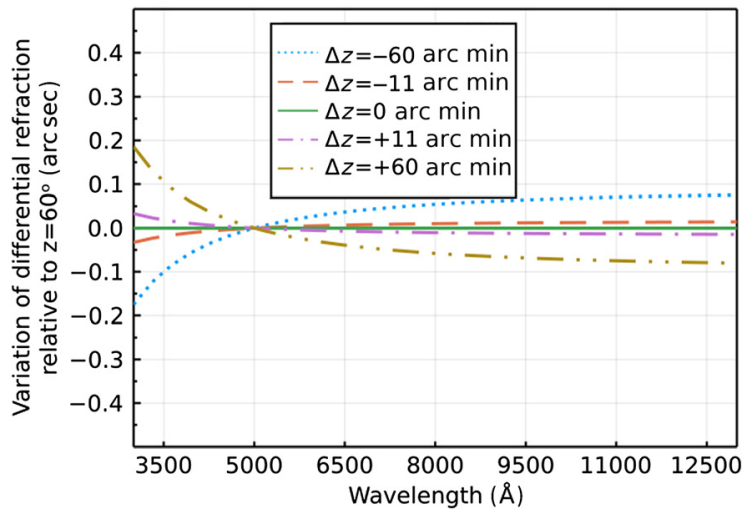

Fig. 2 Variation of the differential refraction across wavelengths at 5 zenith angle offsets in arcminutes with respect to $z=60 \mathrm{deg}$. The refraction at each zenith angle, given with respect to $5000 \AA$, is calculated at 793 mbar in pressure, $15^{\circ} \mathrm{C}$ in temperature, and $2060 \mathrm{~m}$ in elevation.

The telescope field astigmatism generally takes the following form:

$$
\Phi_{\text {tel }}=C H^{2} r^{2} \cos ^{2} \theta=\frac{C H^{2} r^{2}}{2}(1+\cos 2 \theta) \text {. }
$$

$C$ is the astigmatism coefficient given by the telescope design, $H$ is the radial field position normalized to the maximum corrected radial telescope field $\left(H_{\max }\right)$, and $(r, \theta)$ is the normalized polar coordinate on the telescope pupil plane with $r=1$ at the pupil edge. The astigmatism correction at a given $H$ occurs when the total astigmatism given by the cylinder pair $\Phi_{\text {syn }}(H)$ equals $-\Phi_{\text {tel }}(H) . \Phi_{\text {syn }}(H)$ in the collimated beam then can be expressed as

$$
\Phi_{\text {syn }}(H)=f\left(c_{1}, \Delta n_{1}\right) r^{2} \cos ^{2}\left(\theta+\alpha_{1}(H)\right)+f\left(c_{2}, \Delta n_{2}\right) r^{2} \cos ^{2}\left(\theta+\alpha_{2}(H)\right),
$$

where the terms on the right-hand side (RHS) are the astigmatisms from the first and the second cylinder lenses, respectively. The coefficients are given as $f\left(c_{i}, \Delta n_{i}\right)=c_{i} \Delta n_{i} \rho_{0}^{2}$, i.e., functions of the cylinder surface curvature $\left(c_{i}\right)$ and glass index different from air $\left(\Delta n_{i}\right)$ at a chosen wavelength $(\lambda)$. Notice the squared pupil radius $\left(\rho_{0}\right)$ to scale $f\left(c_{i}, n_{i}\right)$ to a proper length unit. $\alpha_{i}(H)$ is the rotation angle of each cylinder with respect to the radial field axis for a unit located at $H$. Depending on which side the cylinder surface faces the air space, $\Delta n_{i}$ can be negative or positive. Assuming the lenses are made of the same materials and both cylinder surfaces facing each other with air gap in between, $c_{1}=c_{2}=c$ since $\Delta n=\Delta n_{1}=-\Delta n_{2}$. Therefore letting $A=c \Delta n \rho_{0}^{2}$ and using the identity of $\cos ^{2} x=\frac{1+\cos 2 x}{2}$, Eq. (2) can be expanded, for arbitrary $\alpha_{1}$ and $\alpha_{2}$, as

$$
\begin{aligned}
\Phi_{\mathrm{syn}}= & \frac{A}{2}\left[\cos 2 \alpha_{1}(H)-\cos 2 \alpha_{2}(H)\right] r^{2} \cos 2 \theta \\
& -\frac{A}{2}\left[\sin 2 \alpha_{1}(H)-\sin 2 \alpha_{2}(H)\right] r^{2} \sin 2 \theta
\end{aligned}
$$

According to Eq. (1), $\Phi_{\text {syn }}(H)$ is proportional to $\cos 2 \theta$, for which $\alpha_{i}$ must be chosen so that, for all $\alpha_{1} \neq \alpha_{2}$ :

$$
\cos 2 \alpha_{1}-\cos 2 \alpha_{2} \neq 0 \quad \text { and } \quad \sin 2 \alpha_{1}-\sin 2 \alpha_{2}=0
$$

By letting $\alpha_{i}(H)=\alpha+\delta_{i}(H)$ with $\alpha= \pm \frac{\pi}{4}, \pm \frac{3 \pi}{4}$ and $\delta(H)=\delta_{1}(H)=-\delta_{2}(H)$, Eq. (4) can be rewritten for $\alpha=\frac{\pi}{4}$ as 


$$
\begin{aligned}
& \cos 2 \alpha_{1}(H)-\cos 2 \alpha_{2}(H)=-2 \sin 2 \delta(H) \\
& \sin 2 \alpha_{1}(H)-\sin 2 \alpha_{2}(H)=0 .
\end{aligned}
$$

Hence, for all $\delta(H)$,

$$
\Phi_{\text {syn }}=-c \Delta n \rho_{0}^{2} \sin 2 \delta(H) r^{2} \cos 2 \theta .
$$

Finally, $\Phi_{\text {syn }}=-\Phi_{\text {tel }}$ leads to

$$
c=\frac{C}{\Delta n \rho_{0}^{2}} \quad \text { and } \quad \delta(H)=\frac{1}{2} \sin ^{-1} H^{2} .
$$

Equation (7) prescribes the curvature and counter-rotation angle of the cylinder across the field. With $c$ as defined above, we need $\delta=\frac{\pi}{4}$ at $H_{\max }$ or $H=1$, hence $\frac{\pi}{2}$ for the total counterrotation angle between two cylinders. With $15 \%$ steeper curvature, we obtain $\delta \leq \frac{\pi}{6}$. Equation (6) can be used to determine the tolerance on $c$ and the control accuracy of $\delta$ at a given $H$. Note that the axially symmetric term in Eq. (1) contributes to the overall telescope field curvature. Although Eq. (6) contains no compensation for this term, if needed, this extra field curvature term can be independently corrected, as part of the overall telescope field curvature, by adjusting the positions of the AWACS units at different radial field positions along their optical axes.

\subsubsection{Cubic plate}

The fourth feature of the AWACS is the item marked as "wedge/cubic plate" in Fig. 1(d). The previous discussion leading up to Eq. (7) considers the point-wise field aberration correction at a specific $H$. Because each AWACS unit has a non-zero FOV, there will be residual astigmatism across the unit FOV (uFOV). This residual astigmatism may or may not be of concern depending on the size of the uFOV, the field astigmatism strength of a telescope, and the tolerance level, all of which are application-specific. To describe the residual astigmatism, Eq. (1) can be expanded around the uFOV center (say $H_{0}$ ) to the linear term of the local field $\delta H$ within the uFOV as

$$
\Phi_{\mathrm{tel}} \approx C H_{0}^{2} r^{2} \cos ^{2} \theta+2 \frac{C \delta H}{H_{\max }} H_{0} r^{2} \cos ^{2} \theta
$$

A cylinder lens pair chosen according to Eqs. (6) and (7) corrects the first term on the RHS of Eq. (8). The second term corresponds to the residual linear astigmatism. At this point, two questions arise. First, "where in the unit should an attempt be made to correct the residual?" Since it is field dependent, a corrective element near the front or back focus of the AWACS unit would work effectively. The second question is, "what would this element look like?" Upon a close look at Eq. (8), the residual term turns out to be a cubic function, i.e., linear in $\delta H$ and quadratic in $r$. Hence, the corrective element should have a cubic surface profile along the radial field axis. With the assumption of this cubic plate at the input side of the AWACS unit, we derive its surface profile as follows.

Suppose that the telescope beam at field $H$ strikes the cubic plate at height $x$ with respect to its center $x=0$. The cubic surface profile at $x$ is given as $F(x)=D x^{3}$, where $D$ is the cubic coefficient. The cubic surface imparts the following optical path length on the chief ray at $H$ :

$$
\Phi_{\text {cubic }}=D \Delta n x^{3} .
$$

For the marginal ray originating from the same field but striking a point offset from the chief ray by $\delta x$, Eq. (9) can be expanded around $x$ as

$$
\Phi_{\text {cubic }}=D \Delta n\left(x^{3}+\delta x^{3}+3 x \delta x^{2}+3 x^{2} \delta x\right) .
$$

The first term in $(\cdot)$ on the RHS of Eq. (10) is a constant offset with no impact on the total aberration of the beam and hence can be ignored. The second term imprints coma aberration, 
but the magnitude may be insignificant for small beam footprint, i.e., small $\delta x$. The third term would manifest as a linear astigmatism over the beam footprint along the $x$ axis and can cancel out the linear term in Eq. (8). Since $x$ is the linear field position with respect to the center of the cubic plate, $x=f_{\text {tel }} \delta H$, where $f_{\text {tel }}$ is the telescope focal length. $\delta x$ is the coordinate within a beam footprint and can be expressed as $\delta x=\frac{l}{2 F} \delta \tilde{x}$ in terms of the beam focal ratio $(F)$ and the axial distance of the cubic plate relative to the beam focus $(l) . \delta \tilde{x}$ is equivalent to the normalized pupil coordinate. At the pupil edge along the $x$ axis, both $r^{2} \cos ^{2} \theta$ and $\delta \tilde{x}$ become unity (since $\theta=0$ ). Equating this term to the linear term of Eq. (8), $D$ can be prescribed as

$$
D=-\frac{8 C F^{2} H_{0}}{3 \Delta n f_{\text {tel }} H_{\max } l^{2}},
$$

which is in the unit of (length/length ${ }^{3}$ ). Equation (11) highlights that $D$ is a function of $H_{0}$ but can stay constant by adjusting $l$ as a function of $H_{0}$. Hence, the same cubic surface may be used to compensate for different amounts of linear astigmatisms at different fields by varying $l$, e.g., shorter $l$ as the cubic plate approaches near the field center where the linear astigmatism is zero. In addition, a higher index glass may help achieving the same degree of correction with a shallower cubic profile, which could alleviate potential difficulty in fabricating the cubic surface.

The last term of Eq. (10) corresponds to wavefront tilt and hence drives the beam away from its nominal angle by the amount varying quadratically in $x$. Such deviation can increase the chief ray angle with respect to the local normal vector on the image surface, which we call telecentricity $\left(\alpha_{\mathrm{tc}}\right)$. In fiber-fed spectrographs, larger telecentricity with respect to the fiber optical axis could result in beam speed faster than the spectrographs are designed for, hence potentially increasing light loss. This is surely an unfortunate side effect of the cubic plate but may turn out to be tolerable depending on applications. Using Eq. (11) and notations used above for $x$ and $\delta x, \alpha_{\mathrm{tc}}$ at the maximum unit field is expressed as

$$
\alpha_{\mathrm{tc}}=3 D \Delta n\left(f_{\mathrm{tel}} h_{\max }\right)^{2}=-\frac{8 C F^{2} H_{0} f_{\mathrm{tel}} h_{\mathrm{max}}^{2}}{H_{\mathrm{max}} l^{2}} .
$$

Equations (11) and (12) can provide a prescription for a given telescope to allow compensating $\alpha_{\mathrm{tc}}$ by optimizing the relay part of the unit design. Though this may result in a loss of generality of the unit relay design, many telescopes would likely have suboptimal native telecentricity and necessitate such a telescope-specific design change to the base relay. As part of such optimization, one could include a wedge angle to the cubic plate to steer the telescope beam at each unit toward more favorable directions in order to achieve not only better gross telecentricity at the output but also the optimal packaging of the units behind the telescope focal surface. Also notice that $\alpha_{\mathrm{tc}}$ depends quadratically on $l$ like $D$, hence the telecentric angle will diminish for those units closer to the telescope field center.

\subsection{Straw-Man AWACS with a Notional Relay}

The aforesaid canonical features are designed here for two telescope cases, one for the $F / 9$ Ritchey-Chrétien (RC) 2.7-m HJST at the McDonald Observatory and the other for a hypothetical F/8 Aplanatic Gregorian 25-m telescope (AGT), similar to the GMT. Note that these telescopes and all AWACS designs presented hereafter have been modeled in OpticStudio ${ }^{\circledR}$ (version 20.2) by Zemax. ${ }^{25}$ The basic characteristics of the telescopes and the corresponding AWACS parameters are listed in Table 1.

An example straw-man design of the HJST-AWACS unit is shown in Fig. 3. The unit is based on a notional relay that contains a pair of cylinder lenses at the center of the collimated space. This lens pair, at the maximum counter rotation angle of $90 \mathrm{deg}$, corrects the incoming telescope astigmatism at 40.2 arc min field. The residual linear astigmatism is corrected by the cubic plate at the front of the relay. The cubic plate is $40 \mathrm{~mm}$ in diameter and $5 \mathrm{~mm}$ thick.

At 40.2 arcmin field, the beam telecentric angle to the HJST's concave focal surface is $2.9 \mathrm{deg}$, but this includes the curvature angle of the focal surface. With respect to the HJST optical axis, the beam angle is $1.4 \mathrm{deg}$, in which the entire unit is tilted to be aligned to the 
Table 1 (Left) Basic telescope parameters and (right) AWACS unit parameters. Astigmatism and coma are field aberrations. The aberration coefficients have been calculated for each telescope in unit of $\lambda=550 \mathrm{~nm}$. FS, fused silica; CC, concave; and CX, convex.

\begin{tabular}{|c|c|c|c|c|c|c|c|}
\hline \multicolumn{4}{|c|}{ Telescope } & \multicolumn{4}{|c|}{ AWACS unit } \\
\hline Parameters & HJST & AGT & Units & Parameters & HJST & AGT & Units \\
\hline Form & $\mathrm{RC}$ & $A G$ & - & Magnification & $1 \times$ & $1 \times$ & - \\
\hline Aperture $\left(D_{\text {tel }}\right)$ & 2.7 & 25.4 & $\mathrm{~m}$ & uFOV & 4.2 & 1.0 & arc min \\
\hline Focal length $\left(f_{\text {tel }}\right)$ & 23.9 & 202.7 & $\mathrm{~m}$ & Focal length & 100 & 150 & $\mathrm{~mm}$ \\
\hline Focal ratio $(F /)$ & $\mathrm{F} / 9$ & $\mathrm{~F} / 8$ & - & $\Delta n(\mathrm{FS})$ & 0.459 & 0.459 & - \\
\hline Plate scale & 0.116 & 0.983 & $\mathrm{~mm}$ & $\rho_{0}$ in Eq. (7) & 5.75 & 9.35 & $\mathrm{~mm}$ \\
\hline Corrected FOV dia. & 84 & 22 & $\operatorname{arc} \min$ & $c^{-1}$ in Eq. (7) & 1144 & 1200 & $\mathrm{~mm}$ \\
\hline Curvature radius & $3.75 \mathrm{CC}$ & $2.2 \mathrm{CX}$ & $\mathrm{m}$ & $\delta$ in Eq. (7) & \pm 45 & \pm 45 & deg \\
\hline Astigmatism & 24.1 & 61.2 & $\lambda$ & I in Eq. (11) & 70 & 70 & $\mathrm{~mm}$ \\
\hline Coma & -0.223 & 2.79 & $\lambda$ & $D$ in Eq. (11) & $4.5 \times 10^{-6}$ & $3.8 \times 10^{-6}$ & $\mathrm{~mm}^{-2}$ \\
\hline Spherical Aberr. & 0.045 & 0.024 & $\lambda$ & $\alpha_{\mathrm{tc}}$ in Eq. (12) & 0.076 & 0.264 & deg \\
\hline
\end{tabular}

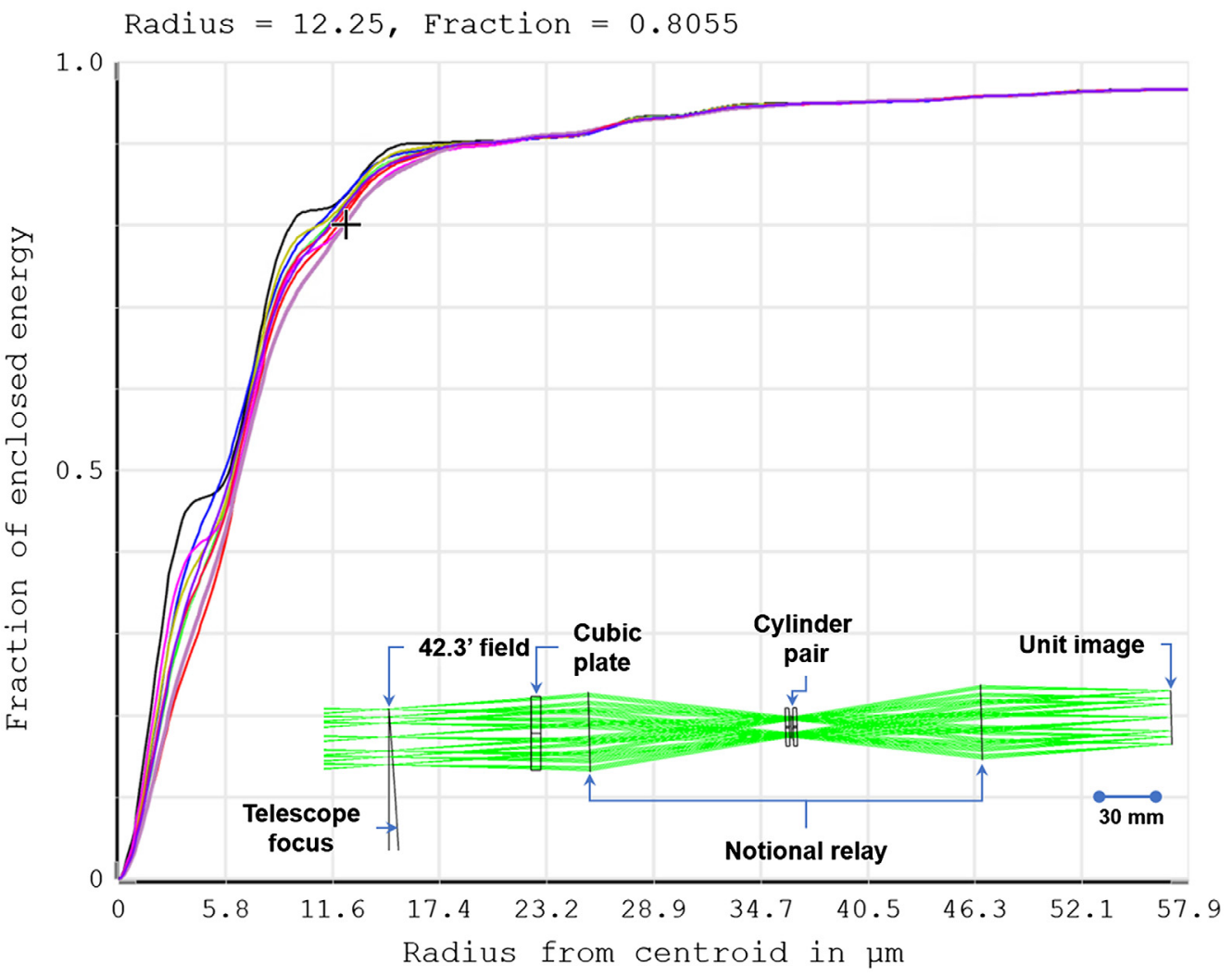

\begin{tabular}{|c|c|c|}
\hline $\begin{array}{l}\mathbf{\square}-\text { Diff. Limit } \\
\mathbf{\square}-0.0000,0.0175 \text { (deg) } \\
\square-0.0175,0.0000 \text { (deg) }\end{array}$ & $\begin{array}{l}\square-0.0000,-0.0350 \quad(\mathrm{deg}) \\
\square-0.0000,0.0350 \quad(\mathrm{deg}) \\
\square-\end{array}$ & $\begin{array}{l}\nabla-0.0000,-0.0175 \text { (deg) } \square-0.0000,0.0000 \text { (deg) } \\
\square--0.0350,0.0000 \text { (deg) } \square--0.0175,0.0000 \text { (deg) } \\
\square-0.0251,0.0251 \text { (deg) } \square--0.0251,-0.0251 \text { (deg) }\end{array}$ \\
\hline
\end{tabular}

Fig. 3 Polychromatic diffraction enclosed energy diagram within the UFOV for a combination of the HJST and a straw-man AWACS unit (based on the HJST parameters in Table 1) shown as centered at 40.2 arcmin field (inset). Each curve represents a different field position in the uFOV. $r_{80} \leq 0.11$ arc sec or $12.2 \mu \mathrm{m}$ (marked by a cross) and near diffraction limit at 0.15 arc sec or $17.4 \mu \mathrm{m}$. The telecentricity is $0.114 \mathrm{deg}$ maximum. Rays propagate from left to right. The uFOV field angles in the legend are given in degrees. 

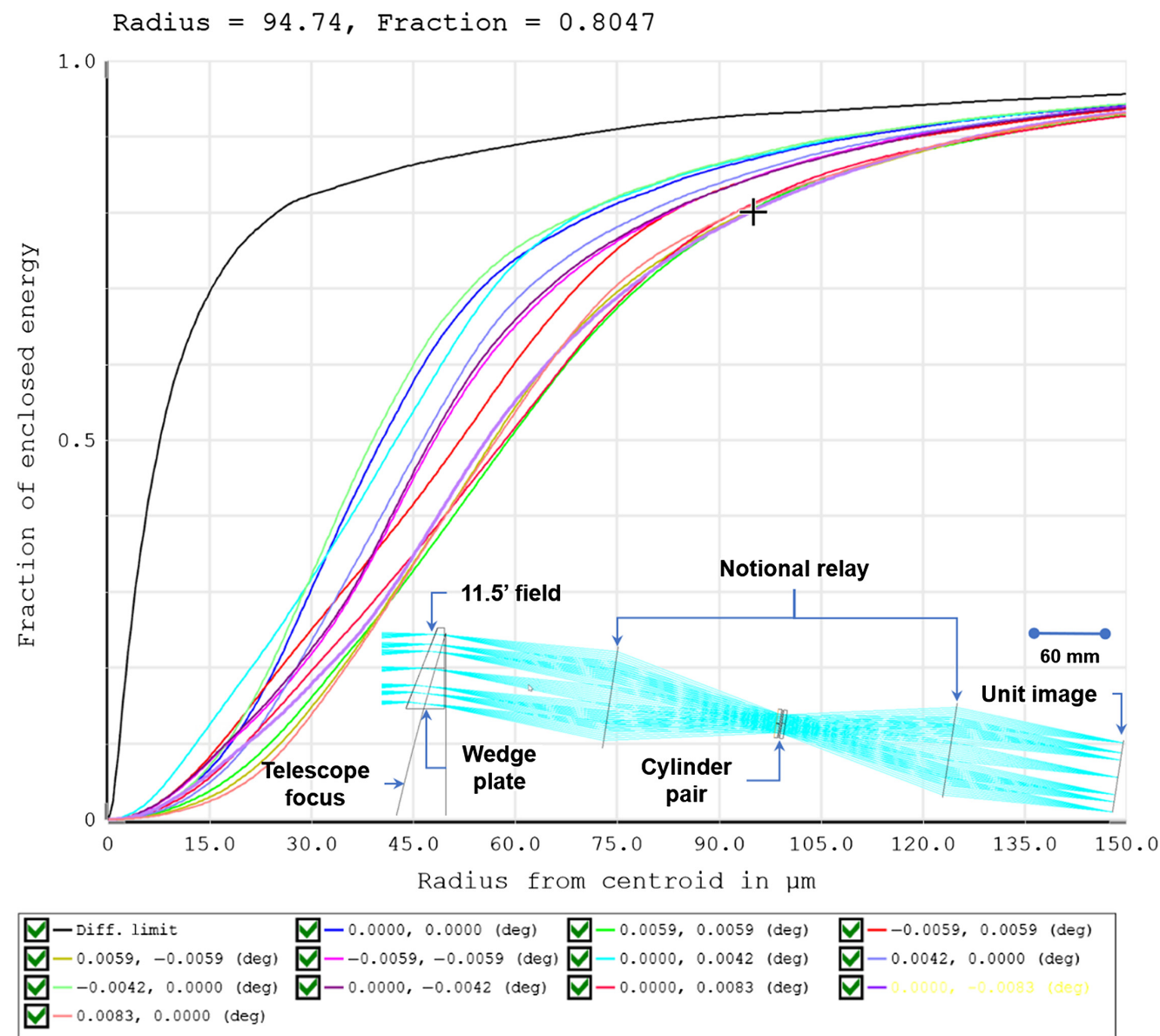

Fig. 4 Polychromatic diffraction enclosed energy diagram within the uFOV for a combination of the AGT and a straw-man AWACS unit (based on the AGT parameters in Table 1) shown as centered at 11 arc min field (inset). Each curve represents a different field position in the uFOV. $r_{80} \leq 0.1$ arc sec or $94.74 \mu \mathrm{m}$ (marked by a cross). The telecentricity is 0.065 deg maximum. In the inset, rays propagate from left to right. The classic Gregorian convex focal surface is evident with rather native telecentric angle of $17 \mathrm{deg}$ at 11 -arc min field. Unlike the HJST case with concave field curvature, a wedge plate is used near the object plane of the AGT-AWACS unit in order to steer the steep beam angle to a direction favorable for imaging performance and packaging. The wedge plate also equalizes the beam path lengths across the uFOV. The image quality without a cubic plate is deemed acceptable in this analysis. The UFOV field angles in the legend are given in degrees.

beam axis. The cylinder and cubic plate parameters in Table 1 were used as the initial seed for further numerical optimization targeting both imaging performance and telecentricity. The optimized parameters are $c^{-1}=1143.9, D=5.2 \times 10^{-6}$, and $2 \delta=90$ deg. $\alpha_{\mathrm{tc}}$ at the edge of the uFOV is $0.114 \mathrm{deg}$. A portion of the telecentric angle comes from the cubic plate in the form of quadratic contribution of $0.091 \mathrm{deg}$. These compare well to those in this table. For any point source over the uFOV, $80 \%$ of diffraction energy is contained within the radius of $r_{80}=0.11$ arc sec. The unit becomes diffraction limited at 0.15 arc sec radius.

Similarly, a straw-man AGT-AWACS design is shown in Fig. 4. The unit is positioned at 11 arc min radial field of the AGT. The AGT's Gregorian convex focal surface is evident with rather extreme native telecentric angle of $17 \mathrm{deg}$ at the field edge. One major difference from the HJST-AWACS is the wedge plate near the object plane instead of a cubic plate. Its main role is to correct the telecentricity by steering the input beam toward a direction more favorable for aberration correction and packaging. It also equalizes the beam path lengths through unequal path within the wedge plate across the uFOV. The straw-man AGT-AWACS reaches $r_{80}$ at $0.1 \mathrm{arc} \mathrm{sec}$ and the diffraction limit near $0.2 \operatorname{arc} \sec ($ or $196 \mu \mathrm{m}$ ) radius from its spot centroid. This is 
deemed acceptable for the sake of this analysis. The telecentric angle to the unit image plane is $<0.065 \mathrm{deg}$, most of which is the residual from the angle adjustment by the wedge plate. Since the wedge plate has no cubic profile, $\alpha_{\mathrm{tc}}$ came out much smaller than the value in Table 1 where a cubic profile is assumed.

\subsection{Example AWACS with Actual Relays and ADC Prisms}

Two HJST-AWACS units with an actual relay and ADC prisms are shown in the inset of Figure 5. One unit shown at the top is centered at 40 arc min radial field and the other at 30 arc min. The polychromatic diffraction enclosed energy diagram for the 40 arc min unit is also shown across the $\mathrm{uFOV}$ at $Z=60 \mathrm{deg}$ with the full ADC. The relay has been designed independently from the telescope. Only the telescope beam speed, uFOV size, and wavelength range have been used in the design process. The relay is color corrected from 350 to $1100 \mathrm{~nm}$. The telescope pupil is imaged to a 6.9-mm diameter disk on the intermediate plane between the cylinder lenses. This indicates the effective focal length of $f_{\text {rel }} \sim 62 \mathrm{~mm}$. The unit, on the HJST, reaches $r_{80}$ at 0.22 arc $\sec ($ or $25 \mu \mathrm{m}$ ) with the maximum telecentric angle of $0.13 \mathrm{deg}$. This corresponds to focal ratio change from $F / 9$ to $F / 8.65$. In the straw-man design (Fig. 3), $r_{80}$ is 0.11 arc sec (or $12.7 \mu \mathrm{m}$ ),

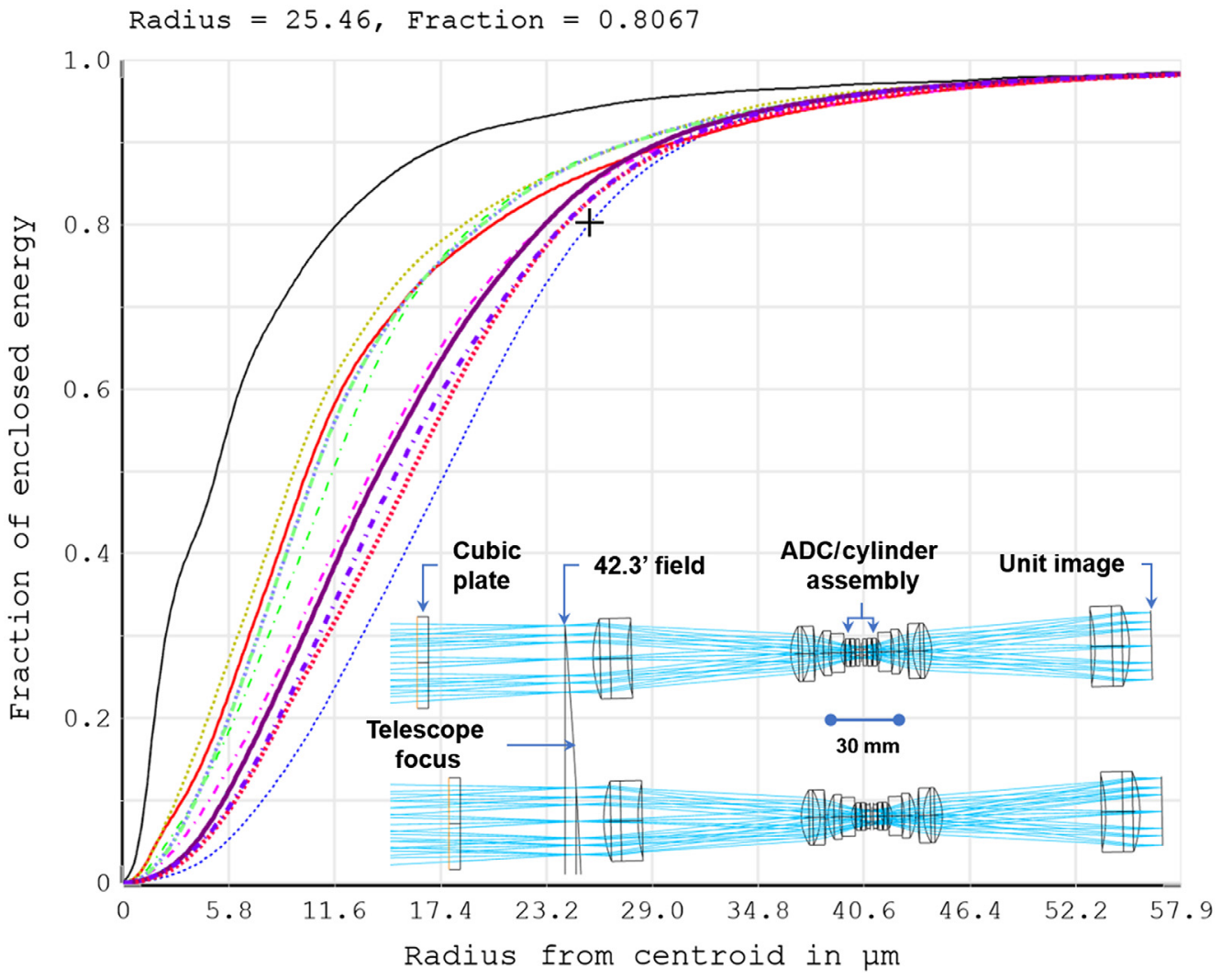

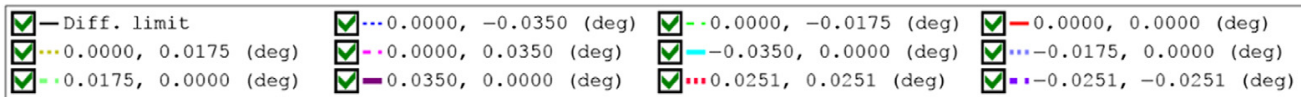

Fig. 5 (HJST-AWACS) Polychromatic diffraction enclosed energy diagram within the uFOV for the combination of the HJST and the field edge unit centered at 40.2 arc min field (top unit in the inset) with $Z=60 \mathrm{deg}$. Each curve represents a different field position in the UFOV. $r_{80} \leq 0.22$ arc sec (marked by a cross) which compares to $r_{80} \leq 0.11$ arc sec for the notional relay in Fig. 3 . The telecentricity is $0.13 \mathrm{deg}$ maximum. This is equivalent to focal ratio change from $F / 9$ to $F / 8.65$. The bottom unit in the inset is centered at 30 arc min. The packing pitch is 10 arc min but can be reduced to 5 arc min without serious issue. Rays propagate from left to right. The uFOV field angles in the legend are given in degrees. 


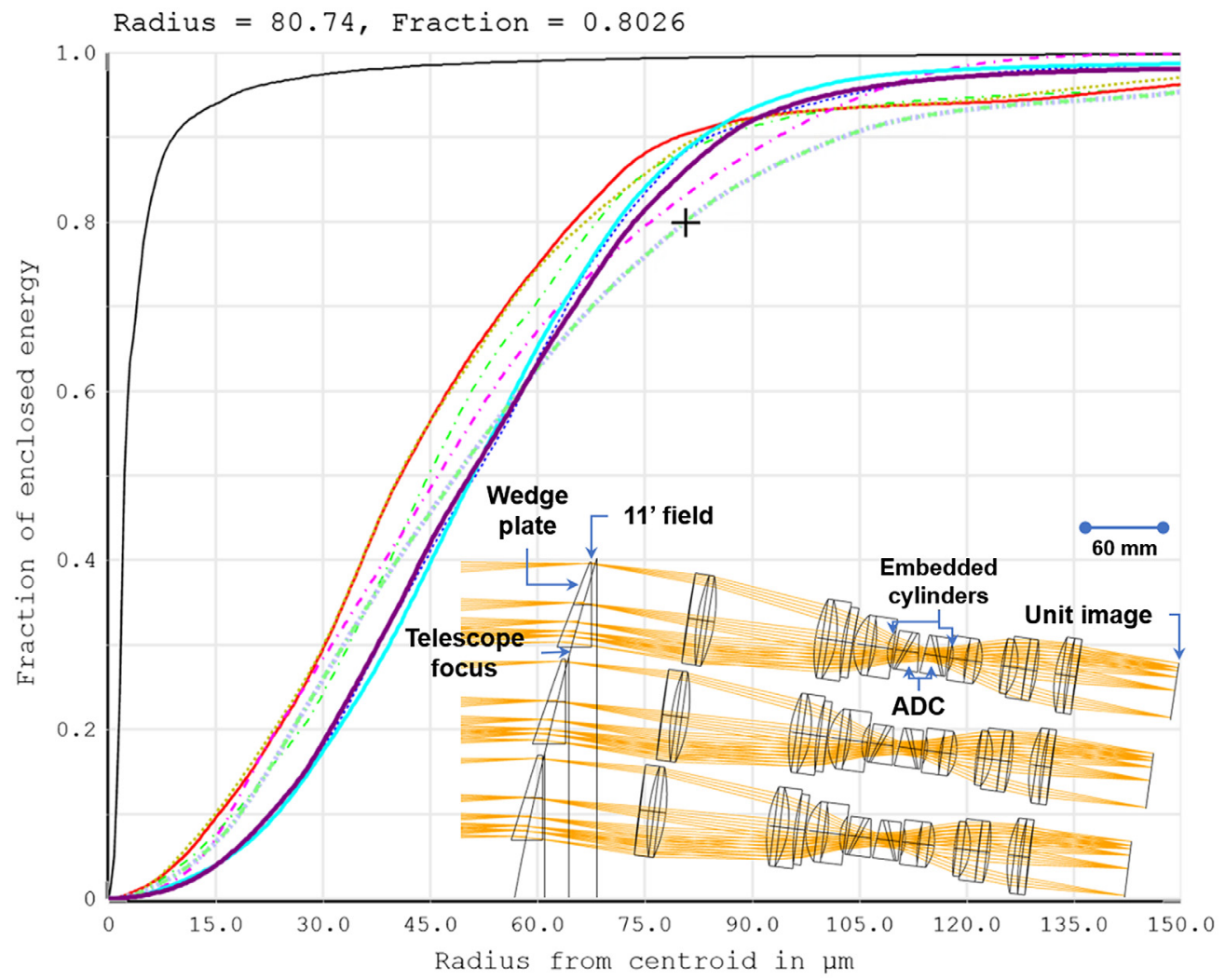

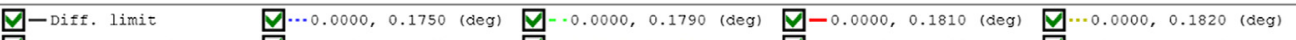
田-0.0000, 0.1833 (deg) $\mathbf{\square}-0.0000,0.1667$ (deg) $\square$...

Fig. 6 (AGT-AWACS) Polychromatic diffraction enclosed energy diagram for the combination of the AGT and the field edge unit centered at 10.5 arc min (top unit in the inset) within the uFOV at $Z=60$ deg. Each curve represents a different field position in the uFOV. $r_{80} \leq 0.11$ arc sec (marked by a cross) which compares to $r_{80} \leq 0.1$ arc sec for the notional relay in Fig. 4 . The telecentricity is 0.13 deg maximum. This is equivalent to focal ratio change from $F / 6$ to $F / 5.84$. The units are shown as closely packaged at 1.25 arc min center-to-center pitch. Rays propagate from left to right. The uFOV field angles in the legend are given in degrees. Note $0.75 \times$ magnification as discussed in the text.

which represents the HJST aberrations and the degree of field correction by the cylinder pair and cubic plate. The difference with respect to the straw-man design comes from the stand-alone performance of this actual unit $(0.17$ arc sec or $19.4 \mu \mathrm{m})$, and imperfect ADC correction $(0.08 \operatorname{arcsec}$ or $9.4 \mu \mathrm{m})$, all in $r_{80}$. Often $50 \%$ energy diameter $\left(d_{50}\right)$ is used instead to evaluate image quality in order to be consistent with the site-seeing metric. For the field edge unit, $d_{50} \leq 0.29$ arc sec across its uFOV. For a typical 1-arc sec site-seeing, this unit would deliver image quality better than $d_{50}=1.04$ arc sec across the corrected 84 arc min HJST field, which is adequate for many observing scenarios.

Figure 6 shows the AGT-AWACS units closely packed with 1.25 arc min center-to-center spacing. The field edge unit is centered at 10.5 arc min radial field. All units are shown with the ADC on. Like the HJST-AWACS, the AGT-AWACS unit relay has been designed for the telescope focal ratio, uFOV, and wavelengths but independent of the telescope aberrations. The unit relay has been color corrected from 350 to $1800 \mathrm{~nm}$. The AGT-AWACS unit images the telescope pupil to a 12-mm diameter disk on the intermediate plane between the ADC prisms. This indicates the unit focal length of $f_{\text {rel }} \sim 96 \mathrm{~mm}$. Since $f_{\text {tel }} / f_{\text {rel }}$ is much greater $(\sim 5 \times)$ for the AGT-AWACS than the HJST-AWACS, the ADC prism surfaces need much steeper angle as discussed in Sec. 2.1.1 and as evident in Fig. 6. The AGT-AWACS ADC air-glass surfaces are angled by $\sim 4 \mathrm{deg}$, whereas it is $\sim 0.9$ deg for the HJST-AWACS. Of course, the exact ADC glass choice influences the prism angle. 
Across the corrected 22 arc min FOV on the telescope, $r_{80}$ remains $<0.12 \operatorname{arcsec}$ at $Z=$ $60 \mathrm{deg}$. One difference of this example from the HJST-AWACS is the choice of $0.75 \times$ magnification, i.e., $F / 8$ at input and $F / 6$ at output, which changes the plate scale from 0.982 to $0.736 \mathrm{~mm} / \mathrm{arc} \mathrm{sec}$. This allows the section of the unit after the second ADC prism to be slimmed down in diameter and facilitates a more aggressive packing of the units behind the convex Gregorian focal surface. Another unique choice of this example design is that the telescope field astigmatism is compensated by the counter rotation of two inner most doublets (instead of a separate pair of cylinder lenses) adjacent to the ADC prisms. Cylinder surfaces are embedded in the back faces of the inner-most doublets as indicated, making them toroidal surfaces. The elimination of four extra surfaces can help increase total throughput of the unit. A likely site for this type of 30-m-class telescopes would have the median-seeing around $0.75 \mathrm{arc} \mathrm{sec}$. For the field edge unit at $Z=60 \mathrm{deg}, d_{50} \leq 0.16$ arc sec and the net delivered image quality would be better than $0.77 \mathrm{arc} \mathrm{sec}$. If the telescope were to be assisted by a ground-layer adaptive optics system, the delivered site-seeing could be improved by $\sim 30 \%$ over $\sim 2 \operatorname{arc} \min ^{26,27}$ or by $\sim 10 \%$ over 5 to 10 arc min. ${ }^{28}$ In these studies, the site-seeing could be corrected to as small as 0.57 arc sec. When combined with the AGT-AWACS field edge unit, the delivered image quality at $Z=60$ deg would be $d_{50} \leq 0.59$ arc sec.

\section{Proof-of-Concept and On-Sky Test Results}

\subsection{Optical Evaluation and Mechanical Integration}

The aberration correction concept by a pair of cylinder lenses has been tested, at a limited level, via a POC AWACS system. Two POCs were built out of off-the-shelf components, resulting in a limited wavelength range and uFOV without ADC prisms. Figure 7 shows the optical layout of the POC unit. The unit consists of 1 -in. diameter commercial optics from Thorlabs. ${ }^{29}$ Two cylinder lenses (Thorlabs LJ4530RM-A and LK4015RM-A) in the collimated space are mounted in manual rotary stages (Thorlabs CRM1). As detailed in Sec. 2.1.2, these cylinder lenses are counter rotated to tune the amount of internal astigmatism. The rotation can be dialed to cancel the telescope astigmatism at different radial fields. We adopted $c^{-1}=458.4 \mathrm{~mm}$ for cylinder lenses as this was the smallest curvature readily available. Also the intermediate pupil size of the POC units was $\rho_{0}=11 \mathrm{~mm}$ due to twice longer $f_{\text {rel }}$ driven by the choices of the commercial lenses. As a result, for the same amount of counter rotation angle, the POC units showed astigmatism correction power by $9 \times$ that of the straw-man design in Fig. 3 and hence, based on Eqs. (6) and (7), the total counter rotation angle at the maximum radial field of 42 arc min would be $6.3 \mathrm{deg}$ rather than $90 \mathrm{deg}$. We note that POC \#2 was located at 30 arc min rather than 40.2 arc

(a)

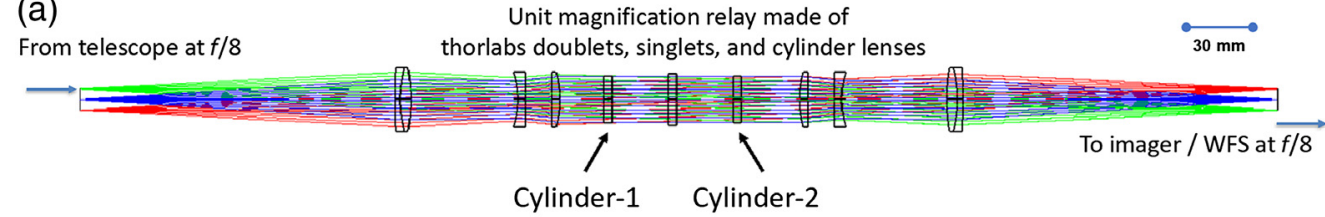

(b)

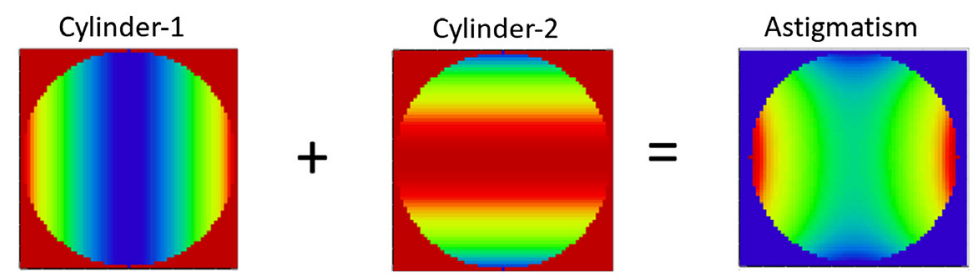

Fig. 7 (a) The POC AWACS unit optical layout consisting of a unit-magnification relay made from commercial optical components. The cylinder lens pair as indicated is mounted in a manual counter rotation mechanism. (b) In the collimated space, two cylinders 90 deg apart in rotation produce pure astigmatic wavefront. For the given cylinder curvature, the counter rotation can be dialed to cancel the astigmatism from the telescope. 


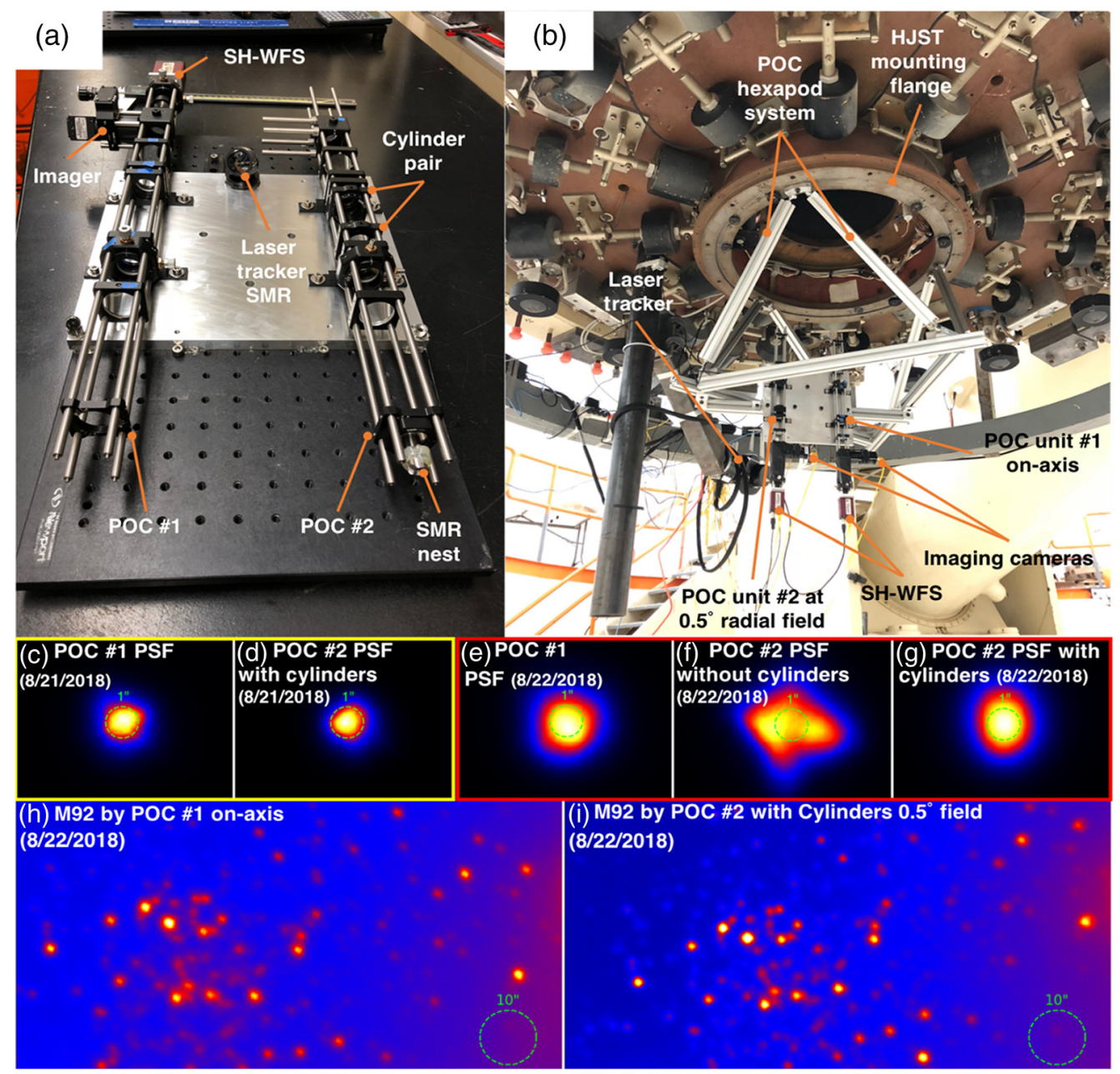

Fig. 8 (a) POC units built out of off-the-shelf components. The POCs had 1.6 arc min dia. FOV and a limited spectral range from 486 to $630 \mathrm{~nm}$. (b) The POCs mounted to the HJST for on-sky test. (c), (d) The 8/21/2018 data where the on-sky PSF image quality in FWHM is 1.1 arc sec (POC 1) and 0.93 arc sec (POC 2). POC 2 had the cylinder lenses to correct the field aberration at 0.5 deg. Dashed circles mark 1 arc sec on-sky. (e)-(g) The 8/22/2018 data where FWHM is 1.3 arc sec (POC 1), 2.2 arc sec (POC 2 without cylinders), 1.4 arc sec (POC 2 with cylinder). (h), (i) M92 images taken on $8 / 22 / 2018$ by POC 1 and 2 . Over the imager field ( 1.6 arc $\min \times 1$ arc $\min$ ), the median FWHM was 1.47 arc sec (POC 1) and 1.4 arc sec (POC 2 with cylinders) with \pm 0.1 arc sec variation in $\mathrm{min} / \mathrm{max}$. A dashed circle on the bottom right corner of each image marks 10 arc sec on-sky.

min radial field due to the HJST flange bore diameter limit. At this field, the counter rotation angle needed to be $3.5 \mathrm{deg}$ rather than $6.3 \mathrm{deg}$. At this counter rotation angle, the POC units produced the standard Zernike astigmatism of $2.7 \mathrm{wv}$ rms (or $13.4 \mathrm{wv}$ in Seidel field astigmatism) that matched the as-designed HJST astigmatism at this radial field position. Without the cylinder pair, the POC units produced total wavefront error $<0.1 \mathrm{wv}$ rms across its designed $\mathrm{uFOV}$ of 1 arc $\min \times 1$ arc min. For the cylinder rotation adjustment, we used a camera setup to visually monitor the physically engraved division marks on the manual rotary stage and wavefront sensor (WFS) feedback. With this ad hoc contraption, we were able to achieve counter rotation angle accurate to $\pm 0.1 \mathrm{deg} \mathrm{rms}$. At this level of angle uncertainty, we estimated the resultant astigmatism to be uncertain by $0.38 \mathrm{wv}$.

Figure 8(a) shows two POC units mounted on a plate. Each unit has been constructed using Thorlabs cage mechanical components and outfitted with a Complementary Metal Oxide Semiconductor (CMOS) imager by Forward Looking Infrared (FLIR) (BFS-PGE-16S7M-C) and a custom Shack-Hartmann WFS via a beam splitting plate (Thorlabs BSW10R) with 50:50 reflection-to-transmission ratio. Notice that we used the cylinder pair only in POC \#2, 
the off-axis unit. The CMOS imager FOV was 1.6 arc $\min \times 1$ arc min on-sky, whereas the WFS FOV was 4 arc sec wide. The front focus of each unit, i.e., where the telescope image forms, has been located by a $50-\mu \mathrm{m}$ diameter pinhole plate. The pinhole was aligned by hand in centration under a microscope with respect to the outer diameter of the lens tube that the pinhole plate was mounted. The pinhole assembly was then positioned to the nominal focus distance with respect to the first lens using feedback from a digital caliper. Given the caliper accuracy (20 to $50 \mu \mathrm{m})$ and machining tolerances of the cage mechanics, we estimate the alignment of the pinhole from the true optical axis of each unit to be better than $250 \mu \mathrm{m}$. The pinhole image relayed to the back focal plane of each unit was used as the optical datum to which the imager and WFS were aligned. The pinhole assembly interfaced each unit's mechanical structure via a magnetic kinematic cage plate (Thorlabs CP44F). This allowed repeatable mount/dismount of the pinhole assembly during alignment on the telescope to $<5 \mu \mathrm{m}$ when measured using our automated precision inc. (API) laser tracker (LT) model T3 in its inteferometric (IFM) mode with 3D coordinate measuring accuracy of $\pm 5 \mathrm{ppm}$. All lab measurement points were within 0.5 -m distance from the LT.

To locate the pinhole in 3D space and thus to allow us to align it to the optical axis and focus of the telescope, we registered the offset location of the center of a spherically mounted retroreflector (SMR) against the pinhole. The SMR was on a separate assembly which interfaced the same kinematic mount. To define the optical axis of each unit, we constructed another point near the back focus. Specifically, we registered the offset location of the center of another SMR with respect to the center of the WFS field stop which is aligned to the relayed pinhole image. The assembly of this SMR threaded directly into the WFS field stop tube. Though this was not kinematic, the repeatability of the SMR to and from the field stop tube was better than $10 \mu \mathrm{m}$. By locating these two SMRs and using their offsets to the pinhole and WFS field stop, we were able to construct the optical axis connecting the object and image side foci of each unit in 3D space. These features on POC \#1 served as the datum for aligning POC \#2 on the same mounting plate to its nominal position and orientation under the LT feedback. After several alignment iterations, we were able to align POC \#2 to POC \#1 to better than $50 \mu \mathrm{m}$ in $x / y / z$ positions and 10 arc sec in tip/tilt. POC \#1 was on-axis and POC \#2 was at 0.5 deg off-axis at the telescope.

On the HJST, the POC plate was mounted to a manual hexpod frame as shown in Fig. 8(b). The frame was then mounted to the telescope's Cassegrain port flange via six 80/20 aluminum extrusion hexapod legs. Spherical bearings were threaded into the ends of each leg. Using two different thread pitches between two bearings of each leg, the hexapod legs acted like a 100 turns-per-inch differential adjuster whose length could be adjusted by as small as $20 \mu \mathrm{m}$. Total range of adjustment was $\pm 5 \mathrm{~mm}$. A set of nuts was placed on each bearings so that they can be tightened to lock the leg length. The telescope mounting flange was used as the surrogate for the telescope optical axis and focus. Therefore, we mounted a set of SMRs on the flange along the circumference. The location of these SMRs constrains the telescope optical axis and focus in 3D space, in which the POC hexapod frame was aligned. We wrote a simple Python script that accepts the current locations of the POC SMRs and returns the leg length changes needed for alignment. Roughly four iterations were needed to complete the alignment. The overall system alignment to the telescope was $\leq 20 \mu \mathrm{m}$ in position and $\leq 5 \mathrm{arc} \mathrm{sec}$ in angle.

For the 3D coordinate measurement of the SMRs, we used the LT in its IFM mode. The LT was mounted on a stainless steel tube drilled into the back of the primary mirror cell to ensure all measurement points to be within 1-m distance from the LT. Prior to the on-sky run, our LT has been cross calibrated against another LT (Faro ION in IFM mode) by measuring a 2-m-long invar calibration rod from various orientations. We verified the IFM mode accuracy to be comparable to the FARO ION LT ( $\pm 5 \mathrm{ppm}$ ) (for FARO LT ${ }^{30}$ and for API $\mathrm{LT}^{31}$ ). At the telescope, we used the same invar calibration rod to verify the IFM mode accuracy of our LT before and after each night during the run. No noticeable change has been detected throughout.

\subsection{On-Sky Test Result}

We were granted four nights on the HJST in late August 2018. The first two nights (August 21 and 22) presented the best opportunity with couple-hours-long clear sky to evaluate the POC AWACS system on-sky. The results are highlighted in Figs. 8(c)-8(i). For each set of measurements, we beamswitched between the units on the same star, with visual magnitude between 
4 and 5. In each set, we took 500 frames through the imagers at 5-ms exposure time. Total three sets of data were taken for each unit. Note that only POC \#2 was equipped with the cylinder lenses. Due to the limitation in the POC system, the telescope was guided in an open-loop fashion by sending pointing offsets roughly every $10 \mathrm{~s}$ to the telescope control system. The offset was estimated from the target image on the POC unit being used. This manual guiding and the resultant telescope pointing drift made it difficult stay the WFS on the target within its 4 arc sec FOV. Also this made it hard to follow through our original plan to adjust the cylinder lens rotation via feedback from the WFS on a on-sky target. As a result, we set the counter rotation of the cylinder lenses based on the WFS measurement of the pinhole target at the front focus of the unit. To turn on the field correction, the counter rotation angle was adjusted until the measured astigmatism equals to the model expectation in which the counter rotation angle was $3.5 \mathrm{deg}$ as done in the lab test.

Figures 8 (c) and 8 (d) show a comparison of delivered image quality by two POC units, using data taken on the night of $8 / 21$. We fit a Moffat function to the target PSF to extract full-width at half-maximum (FWHM), total signal, and Moffat shape parameter. The FWHM of POC \#2 ranged from 0.91 to 0.96 arc sec, across three sets, with median at 0.93 arc sec. The FWHM of POC \#1 ranged from 1 to 1.15 arc sec with median at $1.1 \mathrm{arc}$ sec. The reported DIMM siteseeing was fluctuating between 0.9 and 1.2 arc sec. Across the POC uFOV, the image quality appeared to have stayed at a similar level. This demonstrates excellent field correction by the cylinder lens pair. For the data taken on the night of 8/22 [Figs. 8(e)-8(f)], we first "turned-off" the field correction in POC \#2 by setting the counter rotation angle of the cylinder lenses to 0 deg. As anticipated, this resulted in a significant distortion of the POC \#2 PSF as shown in Fig. 8(f) when compared to that of POC \#1 in Fig. 8(e). The Moffat fit to the POC \# PSF indicated FWHM of 2.2 arc sec. When the correction was turned back on, the POC \#2 PSF quickly morphed, as shown in Fig. 8(g), into a round shape like that in POC \#1 with virtually identical image quality (1.35 arc sec for POC \#1 versus $1.4 \mathrm{arc} \sec$ for POC \#2). Between on and off cases, we observed a substantial elimination of the uncorrected field aberration, amounting to 1.7 arc sec in FWHM. To evaluate the image quality across the POC uFOV, we pointed the units to the central portion of M92 globular cluster. POC \#2 had its cylinder lenses counter rotated to $3.5 \mathrm{deg}$. On each unit, we took 9 frames at 5 -sec exposure. Across all image frames, we picked five bright stars and fit a Moffat function to extract FWHM values. POC \#1 FWHM ranged between 1.44 and 1.57 arc sec, whereas POC \#2 FWHM ranged between 1.38 and 1.46 arc sec. Across the POC uFOV, the image quality variation stayed within $\pm 0.1 \mathrm{arc} \mathrm{sec} \mathrm{level.} \mathrm{We} \mathrm{note} \mathrm{that} \mathrm{the} \mathrm{site-seeing} \mathrm{(median} \mathrm{at} 1.25 \mathrm{arcsec}$ ) was generally poorer than the previous night. Despite the manual guiding hampered wavefront measurements, the image quality as recorded on the imagers demonstrated effective field correction by the cylinder lens pair.

On the POC mechanical system side, the LT measurement verified that the aluminum hexapod system provided $\pm 20 \mu \mathrm{m}$ positioning accuracy and stable alignment of the POC system to the telescope optical axis at the same level across various pointings. The week-long stability measurement indicated that the system stayed within $\pm 50 \mu \mathrm{m}$ with respect to the initial alignment over the temperature swing between $10^{\circ} \mathrm{C}$ and $25^{\circ} \mathrm{C}$, typical west Texas summer. All of these led to no adjustment of the system over 5 days of the on-sky run, highlighting that the integration/alignment processes developed for the POC system is directly transferable to an AWACS on a general two-mirror telescope and much higher quality mechanics will even further increase robustness of such an AWACS.

\section{Antireflective Treatment of Air-Glass Interfaces}

As apparent in Figs. 5 and 6, the AWACS unit contains a large number of air-glass interfaces. The model in Fig. 6 contains 18 such interfaces including 12 in the relay lenses (where 2 cylinder surfaces are embedded), 4 in the ADC prisms, and 2 in the wedge plate. This number could be further reduced using clever optical design techniques or features, but still it would most likely be higher than 15 surfaces. That is a lot of air-glass interfaces where only a small increase in Fresnel reflection loss can quickly add up to significantly undercut the total system throughput. 

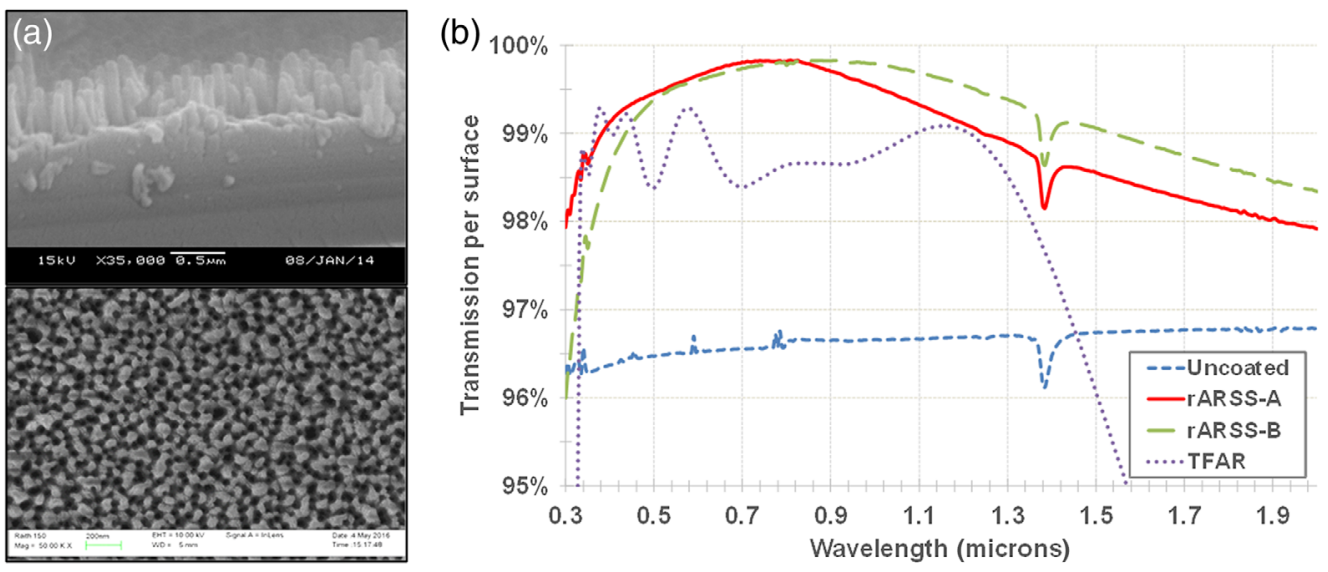

Fig. 9 (a) SEM images of rARSS on flat FS substrates viewed from side (top) and top (bottom). (b) Spectral transmission per surface for two (solid red and green long-dashed) differently processed FS windows, with rARSS on both surfaces, under normal incidence conditions. Transmission curves of uncoated (blue short-dashed) and one of the best TFAR coating (purple dotted) are plotted as comparison.

Hence, it is of paramount importance to suppress reflectivity from these interfaces across a broad wavelength range, especially at near ultra-violet wavelengths.

For these reasons, we have been exploring the use of randomly nano-textured antireflective surface structures (rARSS) on the AWACS air-glass interfaces. These structures are fabricated directly on optical surfaces by a "subtractive" reactive ion-etching process and have been shown to considerably reduce Fresnel reflectivity. ${ }^{32,33}$ Figure 9 (a) shows scanning electron microscope images of a rARSS on a fused silica (FS) window surface. The presence of the structures on optical interfaces results in measurable spectral and directional AR properties. Figure 9(b) shows the single-surface transmission of two differently processed rARSS on FS windows. The curves are computed from silica window measurements of total power transmission along the specular direction (on-axis) of propagation, and thus quantify actual transmittance, not reflectance suppression.

In general, there are three interactions between light wavefronts and optical index boundaries: transmission, reflection, and scatter. Transmission $(T)$ and reflection $(R)$ are due to the discontinuous change of index at the boundary separating the two media, whereas scatter is due to surface structure factors. For transmissive components, reflection and scatter are considered "losses" as they reduce power transfer along the desired direction of propagation and can be combined to a single transmission extinction quantity:

$$
A\left(k, n, \sigma, \theta_{i}\right)=R_{F}\left(n, \theta_{i}\right)\left(1-e^{-\left(2 k \sigma \cos \theta_{i}\right)^{2}}\right)+T_{F}\left(n, \theta_{i}\right)\left(1-e^{-\left(k \sigma|1-n| C\left(\theta_{i}\right)\right)^{\delta}}\right),
$$

where $R_{F}$ and $T_{F}$ are, respectively, the Fresnel reflectance and transmittance power ratios, $k$ is the free-space wavenumber, $\sigma$ is the root-mean-square (rms) roughness of the surface, and $C\left(\theta_{i}\right)$ is the surface structure autocorrelation function. ${ }^{34,35}$ The rms roughness value is associated with the surface structure's depth, whereas $C\left(\theta_{i}\right)$ is a consequence of the transverse dimensions of the surface roughness, which depends on the random structure density and the average crossectional dimension of the random feature populations. All terms depend on the local incidence angle $\theta_{i}$. In this surface model, incident wavelengths longer than the range of values for $\sigma$ and $C\left(\theta_{i}\right)$ incur lower extinction losses, whereas considerable specular transmission losses occur at shorter wavelengths. In Eq. (13), the incident medium (i.e., superstrate) has a refractive index value of 1, and the transmission medium (i.e., substrate) of $n$. Although scalar theory requires the exponent $\delta$ to be equal to 2 , it is empirically fitted to higher values from experimental results, which produce consistent modelling predictions.

Conceptually, the rARSS can be modeled as a surface layer of limited thickness, bridging the optical index transition from the superstrate to the substrate. When the thickness of such a surface layer is similar to the incident wavelength, the transition can be described by effective- 
medium approximations (EMA), such as the Bruggeman EMA. ${ }^{36}$ Within the surface layer, the rARSS can be divided to a number of strata, each of which are subwavelength in thickness and incremental in index, similar to a gradient-index-medium model, which have fully correlated scattering properties, and match the impedance of the electromagnetic wave transition between ambient (incident) and substrate (transmitted) regions. The gradient-index EMA then can predict the degree of suppression of specular reflectivity and losses in the forward and reverse direction due to the rARSS surface layer.

The process used to fabricate the rARSS on FS substrates involves partial-vacuum reactiveion plasma etching, in the presence of fluorine gaseous radicals. The process is directed by an RF-field, which performs ballistic (sputtering) as well as adsorptive (etching) processes concurrently. The substrates are precleaned and coated with a porous gold (Au) thin film, acting as a "seed" to the random etching process. Balancing the process-controlled parameters results in a dense, randomly etched surface, with all the Au removed. The substrates are cleaned further, if needed, with an oxygen rich plasma. ${ }^{37}$

When a single-sided rARSS FS substrate is compared to a plain bare FS window, its as-measured forward scattering distributions at $633 \mathrm{~nm}$ is negligible. Specifically, total integrated scatter of the rARSS surfaces is lower than eight-orders of magnitude in transmission with respect to the nominal transmission across the visible and NIR bands. ${ }^{38,39}$ In addition, due to the randomness and subwavelength scale of the transverse rARSS features, periodic surface-structure scatter effects are also eliminated. This is a fundamental difference between rARSS and subwavelength notch-filter gratings, which can also be used for limited waveband reflectivity suppression. ${ }^{40}$ Randomness also reduces polarization effects due to the angle-of-incidence on surfaces. Reported results show indistinguishable performance in enhanced transmission for an FS window with rARSS, from normal incidence to Brewster's angle. ${ }^{41}$ For angles of incidence (AOI) within $\pm 15 \mathrm{deg}$, there is no shift of the spectral transmission characteristics. Variable AOI tests at $633 \mathrm{~nm}$ wavelength show insensitivity to polarization and AOI. The variable AOI spectral measurements are a topic of investigation in our on-going development. Two-surface transmittances exceeding $99.0 \%$ on FS windows, lenses, micro-lens arrays, and gratings have also been reported..$^{32,41-43}$

Measured rARSS performance from two separately processed, 1-mm-thick, FS slab surfaces (Corning 7980/UV-grade) is shown in Fig. 9(b). The transmission per surface structured is $\geq 99 \%$ from $380 \mathrm{~nm}$ (rARSS-A) to $1550 \mathrm{~nm}$ (rARSS-B). Substrate rARSS-A has enhanced transmission at shorter wavelengths, and substrate rARSS-B shows higher transmission toward longer wavelengths. Of note is the featureless spectral transmission profile, when compared to one of the best-effort thin-film AR (TFAR) coatings, which are limited to a narrower spectral bandwidth with distinct interference features, nonideal for spectroscopic applications. The width of the bandpass and the short-wavelength cutoff edge, due to scattering from the surface structures, can be "tuned" by the fabrication process parameters. Physically, the rARSS is formed on the substrate itself, resulting in similar physical properties to that of the bulk material. The structures have been shown to perform well under environmental and erosive tests. ${ }^{44,45}$

For a system-level comparison, we constructed, as in Fig. 10, throughput models of the HJST-AWACS (Sec. 2.2) using some of the state-of-the-art AR coating options. We made our best effort to consider as many effects as possible, including the glass bulk transmission, scattering loss, and cement loss based on our own experiences. Individual coating options may look similar on the per-surface basis, but because of many air-glass interfaces, a minute difference can make a noticeable impact on the total throughput. The rARSS, as it currently is, shows featureless spectral responses compared to the other models and generally much higher throughput with wide $\Delta \lambda(\sim 600 \mathrm{~nm}$ at $90 \%$ and $\sim 1000 \mathrm{~nm}$ at $80 \%$ level). Achieving even higher throughput over broader $\Delta \lambda$ seems promising with further development. We note that the multi-layer BB TFAR coating tends to require more film stacks, which causes strong coating stress, that needs to be carefully balanced to avoid coating failure and amplify sensitivity to angle of incidence. Sol-gel is an interesting option. It works similarly as the rARSS, but is different in a way that the sol-gel process is "additive" by building on a substrate a surface layer of micro-scale silica particles randomly trapped in a porous gelatinized colloidal solution film either spin-coated or dipcoated. ${ }^{48}$ In the early days, it was known to exhibit extreme fragility to temperature, moisture, and abrasion. Most recent developments improved its environmental robustness and adhesion to 


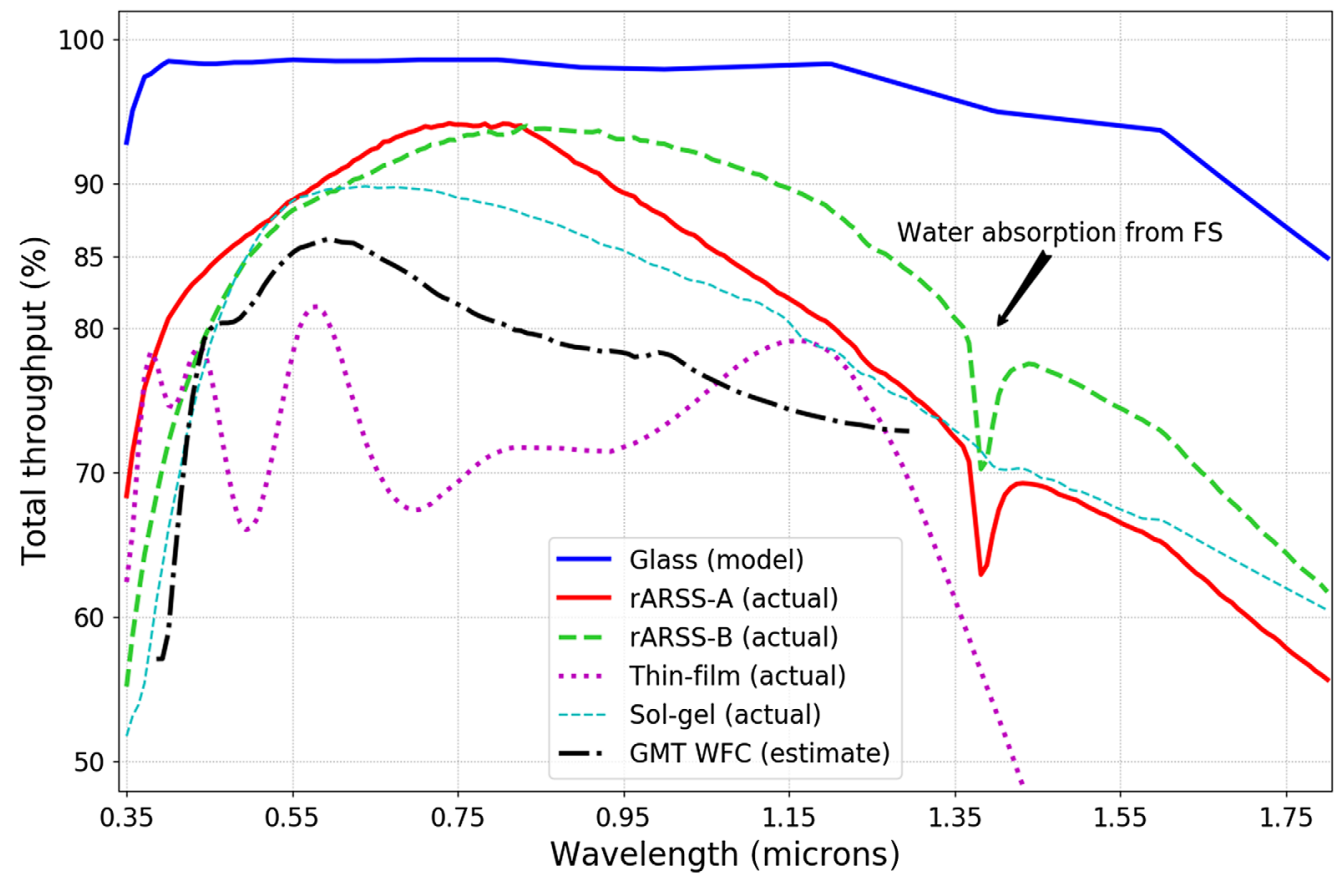

Fig. 10 AWACS throughput models for different AR options. The "glass" sets the highest throughput possible given by the internal transmission of the OHARA i-line glasses used in the HJSTAWACS design in Zemax. Two rARSS transmission measurements from Fig. 9(b) are used which already includes scattering loss. We included the optical cement transmission $(0.1 \%$ loss per cement) to the rARSS-based throughput models. The same is applied to the thin-film model that is based on an actual measurement hence includes scattering loss. The sol-gel type AR coating model additionally includes scattering loss (equivalent to 1.5 -nm rms surface $\mu$-roughness) since this AR model is an actual measurement. ${ }^{46}$ The GMT WFC estimate is shown as a comparison. This model is derived from dividing the DGWF curve (i.e., at the direct Gregorian wide-field focus, hence telescope + wide-field system throughput) by the DGNF curve (i.e., at the direct Gregorian native focus, hence telescope throughput only) in Figs. 4-3 of this technical document ${ }^{47}$ ).

substrate. ${ }^{46}$ In the throughput model plot, the rARSS outperforms the sol-gel AR coating in this comparison, but both options appear mature enough to be applicable to the AWACS optics.

\section{Conclusion}

We detailed the architectural features, design examples, on-sky demonstration, and super broadband AR technologies of the AWACS in the context of ELT-class astronomical wide-field systems. With more in depth discussion of aberration theories behind the choice of optical design features and of the working mechanisms of the rARSS, this paper significantly expands our previous reports ${ }^{49-51}$ on the AWACS architecture. Although there are overlaps, at conceptual levels, with the previous studies, ${ }^{17,18}$ the presented work extends far beyond the concept into more practical and important considerations pertaining to the actual design, prototyping, and construction of such a system. We believe this is the unique contribution of this paper to the field of astronomical telescopes, instruments, and systems.

As discussed, the AWACS accomplishes desired field expansion by operating simultaneously an array of small cost-effective EOM units over a telescope's focal surface. Together with the design commonality across the units, this makes the AWACS orthogonal to the traditional field corrector design approach at both fundamental and practical levels. When such characteristics are optimally implemented, the AWACS can potentially promise economic and technical advantages that would enable controlling the risk and cost of such a system within a much manageable limit, independent of whether it is the HJST, a 25-m AGT, or a 100-m telescope. Our POC on-sky test demonstrated not only the feasibility of the local field aberration compensation, but also 
what can be achieved in terms of optomechanical robustness with more refined design and implementation of the AWACS EOM system.

We anticipate another on-sky demonstration with fully functional science grade EOM units based on our on-going AWACS-Duo project for the HJST in the very near future. ${ }^{51}$ Developing a super compact electro-mechanical ADC rotary stage ${ }^{52}$ and implementing rARSS on some of the optical components are the additional key aspects of this effort. In addition, new optical relay designs are under development to cut down the number of elements to $\leq 5$ from what is presented. This will be an important step toward not only improving the overall transmission but also opening up spectral bandpass below $350 \mathrm{~nm}$ to enable additional sciences on these telescopes, such as abundance analysis of extremely metal poor stars. We pay a special attention to the rARSS because of its potential benefit in much larger groups of electro-optical devices, particularly those subject to harsh-environment such as high-power laser, cryogenic, and space systems. The recent advancement in the free-form optics fabrication now makes it feasible and more economical to build precision cubic plates needed for some versions of the AWACS. Nearly automated fabrication and metrology of standard optics, particularly in diameters ranging from $1 / 2$ to 4 in., allows routine high-volume production of precision spherical, aspheric, and flat optical elements with a strong unit cost scaling, which is needed for replicating the AWACS units.

The presented designs, on-sky test, and availability of key technologies are strong indication of the AWACS as a viable wide-field architecture for the ELTs. With the continuous advances in these relevant areas, the AWACS seems uniquely positioned to enable, either by itself or by complementing traditional monolithic field correctors, wide-field multi-object spectroscopic surveys in the era of the ELTs and beyond.

\section{Acknowledgments}

This work was supported by the McDonald Observatory. Portion of the on-sky data postanalysis effort has been supported by the Mt. Cuba Astronomical Foundation. Hanshin Lee is grateful for Nouf Alabdullatif at the Emory University and Faisal Aalouh at the University of Wisconsin for their contribution to constructing the POC units in summer 2018. Hanshin Lee also would like to thank Brian Vattiat, John Good, Coyne Gibson, Darrin Crook, and Kevin Meyer at the McDonald Observatory for their contributions to the mechanical design and engineering run of the presented work. The authors have no relevant financial interests in the manuscript and no other potential conflicts of interest to disclose.

\section{References}

1. GMT Science Advisory Committee and GMT Project Office, The Giant Magellan Telescope Science Book 2018, GMTO Corporation, Pasadena, CA (2018).

2. W. Skidmore, "Thirty meter telescope detailed science case: 2015," Res. Astron. Astrophys. 15, 1945-2140 (2015).

3. E-ELT Science Office and E-ELT Science Working Group and Science Teams of the Consortia of the E-ELT Instrument Concept Studies, "An expanded view of the universe: science with the European extremely large telescope," European Southern Observatory, Munchen, Germany (2015).

4. M. Colless et al., "The 2dF Galaxy Redshift Survey: spectra and redshifts," Mon. Not. R. Astron. Soc. 328, 1039-1063 (2001).

5. M. R. Blanton et al., "Sloan Digital Sky Survey IV: mapping the Milky Way, nearby galaxies, and the distant universe," Astron. J. 154, 28 (2017).

6. D. J. Eisenstein et al., "SDSS-III: massive spectroscopic surveys of the distant universe, the Milky Way, and extra-solar planetary systems," Astron. J. 142, 72 (2011).

7. J. A. Kollmeier et al., "SDSS-V: pioneering panoptic spectroscopy," arXiv:1711.03234 (2017).

8. K. Hawkins et al., "Using chemical tagging to redefine the interface of the Galactic disc and halo," Mon. Not. R. Astron. Soc. 453, 758-774 (2015). 
9. S. R. Furlanetto, M. McQuinn, and L. Hernquist, "Characteristic scales during reionization," Mon. Not. R. Astron. Soc. 365, 115-126 (2006).

10. N. A. Grogin et al., "CANDELS: the cosmic assembly near-infrared deep extragalactic legacy survey,” Astrophys. J. Suppl. Ser. 197, 35 (2011).

11. S. L. Finkelstein et al., "A galaxy rapidly forming stars 700 million years after the big bang at redshift 7.51," Nature 502, 524-527 (2013).

12. S. C. Odewahn et al., "VIRUS parallel observations with the Hobby-Eberly telescope," in Am. Astron. Soc. Meeting Abstracts, Vol. 219, p. 424.18 (2012).

13. V. Y. Terebizh, Survey Telescope Optics, SPIE Press, Bellingham, WA (2019).

14. M. R. Ackermann et al., "Exploration of wide-field optical system technologies for sky survey and space surveillance," in the 30th Space Symp., Technical Track, Colorado Springs, Colorado, pp. 1-28 (2014).

15. H. Lee et al., "Delivery, installation, on-sky verification of the Hobby-Eberly telescope wide field corrector," Proc. SPIE 9906, 990646 (2016).

16. M. R. Ackermann, J. T. McGraw, and P. C. Zimmer, "Improved spherical aberration corrector for fast spherical primary mirrors," Proc. SPIE 7061, 70610I (2008).

17. J. H. Burge and J. R. P. Angel, "A 30-m Cassegrain telescope with spherical optics and a 3-deg. field," Proc. SPIE 4840, 574-582 (2003).

18. R. Ragazzoni et al., "A smart fast camera," Proc. SPIE 5492, 121-127 (2004).

19. G. J. Hill et al., "Deployment of the Hobby-Eberly telescope wide-field upgrade," Proc. SPIE 9906, 990606 (2016).

20. S. J. Medwadowski, "Structure of the Keck telescope—an overview," Astrophys. Space Sci. 160, 33-43 (1989).

21. R. A. Perley et al., "The expanded very large array: a new telescope for new science," Astrophys. J. 739, L1 (2011).

22. P. Llull et al., "Characterization of the aware 40 wide-field-of-view visible imager," Optica 2, 1086-1089 (2015).

23. R. N. Wilson, Reflecting Telescope Optics I: Basic Design Theory and its Historical Development, Springer-Verlag, Heidelberg, Germany (1996).

24. R. C. Stone, "An accurate method for computing atmospheric refraction," Pub. Astron. Soc. Pac. 108, 1051-1058 (1996).

25. www.zemax.com.

26. C. Baranec et al., "On-sky wide-field adaptive optics correction using multiple laser guide stars at the MMT," Astrophys. J. 693, 1814-1820 (2009).

27. M. Hart, N. M. Milton, and C. Baranec, et al., "A ground-layer adaptive optics system with multiple laser guide stars," Nature 466, 727-729 (2010).

28. M. A. van Dam, A. H. Bouchez, and B. A. McLeod, "Wide field adaptive optics correction for the GMT using natural guide stars," in Adaptive Optics System IV, Vol. 9148, pp. 386-397, International Society for Optics and Photonics (2014).

29. www.thorlabs.com.

30. "FARO ION features benefits \& technical specification," www.faro.com/laser-tracker (2013).

31. "API product specification tracker3 15-40-60," http://apisensor.com.br (2009).

32. L. E. Busse et al., "Anti-reflective surface structures for spinel ceramics and fused silica windows, lenses and optical fibers," Opt. Mater. Express 4, 2504-2515 (2014).

33. Q. Yang et al., "Antireflection effects at nanostructured material interfaces and suppression of thin-film interference," Nanotechnology 24, 235202-235208 (2013).

34. D. Dominé et al., "Modeling of light scattering from micro- and nanotextured surfaces," J. Appl. Phys. 107, 044504 (2010).

35. J. E. Harvey, Understanding Surface Scatter: A Linear Systems Formulation, SPIE Press, Bellingham, WA (2019).

36. D. Stroud, "The effective medium approximations: some recent developments," Superlattices Microstruct. 23, 567-573 (1998).

37. A. Peltier et al., "Control of spectral transmission enhancement properties of random antireflecting surface structures fabricated using gold masking," Proc. SPIE 10115, 101150B (2017). 
Lee and Poutous: Arrayed wide-field astronomical camera system for spectroscopic surveys...

38. D. A. Gonzalez et al., "Bi-directional scatter and single-surface reflectivity of random antireflective nanostructured surfaces," in IEEE 16th Int. Conf. Smart Cities: Improving Quality Life Using ICT IoT and AI, pp. 210-212 (2019).

39. D. A. Gonzalez and M. K. Poutous, "Bidirectional scattering distribution function of random antireflective nano-roughened surfaces," Proc. SPIE 11682, 1168215 (2021).

40. C.-H. Chang et al., "Nanostructured gradient-index antireflection diffractive optics," Opt. Lett. 36, 2354-2356 (2011).

41. A. Peltier et al., "Polarization insensitive performance of randomly structured antireflecting planar surfaces," Opt. Eng. 57, 037109 (2018).

42. K. Kunala and M. K. Poutous, "Diffraction efficiency performance of random anti-reflecting subwavelength surface structures on prefabricated fused silica binary gratings," Appl. Opt. 57, 4421-4427 (2018).

43. A. P. Eckart, "Optical performance of random antireflection structured surfaces on nonvitreous substrates and fused silica microlens arrays," $\mathrm{PhD}$ Thesis, The University of North Carolina at Charlotte (2018).

44. L. E. Busse et al., "Harsh environment tests of random antireflective surface structures on optics," in Conf. Lasers and Electro-Opt., JTu5A.124, Optical Society of America (2017).

45. D. A. Boyd et al., "Modification of nanostructured fused silica for use as superhydrophobic, IR-transmissive, anti-reflective surfaces," Opt. Mater. 54, 195-199 (2016).

46. A. C. Phillips et al., "Progress toward high-performance reflective and anti-reflection coatings for astronomical optics," Proc. SPIE 7018, 70185A (2008).

47. G. Angeli et al., "GMT Observatory Architecture Document: GMT level-3 requirements document," Tech. Rep. GMT-REQ-03215, Giant Magellan Telescope Observatory Corporation (2018).

48. J. R. Stilburn, "High-efficiency sol-gel antireflection coatings for astronomical optics," Proc. SPIE 4008 (2000).

49. H. Lee, "Arrayed wide-angle camera system (AWACS) for the extremely large telescopes," in Opt. Design and Fabrication (Freeform, IODC, OFT), ITh1B.7, Optical Society of America (2017).

50. H. Lee et al., "Arrayed wide-angle camera system for the extremely large telescopes," Proc. SPIE 10702, $107021 \mathrm{Z}$ (2018).

51. H. Lee et al., "Arrayed wide-angle camera system for wide field imaging and spectroscopy on ELTs: proof-of-concept on-sky test results on McDonald Observatory $2.7 \mathrm{~m}$ telescope," Proc. SPIE 11445, 114453W (2020).

52. H. Lee and B. L. Vattiat, "Ultra-sonic super compact rotary stage for arrayed wide-angle camera system: prototype results," Proc. SPIE 11451, 114514U (2020).

Biographies of the authors are not available. 